\title{
Review
}

\section{In vitro and in vivo effects of HIV protease inhibitors on apoptosis}

\author{
AD Badley ${ }^{\star, 1,2}$ \\ 1 Translational, Immunology and Biodefense Program, Mayo Clinic College of \\ Medicine, Rochester, MN, USA; \\ 2 Division of Infectious Diseases, Mayo Clinic College of Medicine, Rochester, \\ MN, USA \\ * Corresponding author: AD Badley, Mayo Clinic College of Medicine, 200 First \\ Street, SW, Rochester, MN 55905, USA. Tel: + 1507255 6482; \\ Fax: + 1507255 7767; E-mail: badley.andrew@mayo.edu
}

Received 02.11.04; revised 28.12.04; accepted 30.12.04; published online 11.3.05 Edited by $\mathrm{G}$ Kroemer

\section{Abstract \\ Development of potent inhibitors of HIV protease has revolutionized the treatment of HIV infection. HIV protease inhibitors (PI) have caused more dramatic improvements in CD4 T-cell numbers than in other therapies that were available previously, prompting investigators to assess whether PI possess intrinsic immunomodulatory effects. An emerging body of data indicates that HIV PIs are antiapoptotic, although the exact molecular target responsible for this antiapoptotic effect remains to be defined in vitro and in vivo. Paradoxically, high-dose PI also may have proapoptotic effects, particularly when assessed in vitro in transformed cell lines and implanted mouse models. Future research will define molecular targets of PI that are responsible for their apoptotis modulatory effects (both pro- and anti-apoptotic). In addition, evaluation of the clinical utility of PI-based therapy in those non-HIV disease states that are characterized by excessive apoptotis will reveal the full clinical potential of this intriguing class of drugs. \\ Cell Death and Differentiation (2005) 12, 924-931. \\ doi:10.1038/sj.cdd. 4401580 \\ Published online 11 March 2005}

Keywords: proteases; HIV; CD4

Abbreviations: $\mathrm{PI}$, protease inhibitors

\section{Role of HIV Protease in HIV Pathogenesis}

The HIV genome consists of a single-stranded positive sense RNA molecule that is organized into three major coding elements: gag, pol, and env. The enzymes necessary for viral replication (protease, reverse transcriptase, and integrase) are included within the $\mathrm{pol}$ gene. During viral assembly and maturation, P55 gag precursor and P160 gag/pol precursors are protealitically cleaved by preformed protease, which is contained within the HIV virion and introduced into the cell at the time of initial infection. This preformed protease consequently generates new protease by virtue of new cleavages between the protease and reverse transcriptase regions of the pol gene. HIV protease is critical to the HIV life cycle, as demonstrated by observations that mutation or inhibition of HIV protease results in the formation of structurally disorganized, noninfectious viral particles. Moreover, naturally occurring mutations that arise as a consequence of subsuppressive antiretroviral therapy results in generation of viral particles, which have impaired replication kinetics as well as impaired cleavage kinetics of mutant protease against the wild-type gag/pol substrate cleavage site.

In addition to its role in viral replication, HIV protease may also contribute to HIV pathogenesis itself. HIV protease is intrinsically cytotoxic and leads to death of both human and bacterial cells following transfection. In different contexts, the phenotype of HIV protease-mediated death is either apoptotic or necrotic. The mechanisms of cell death by HIV protease remain undefined, although HIV protease itself may directly cleave cell regulatory proteins to favor cell death. For example, Strack and co-workers ${ }^{1,2}$ identified BCL-2 as a apoptosis regulatory protein, which is cleaved by HIV protease. More recently, other cellular proteins, including actin, laminin-B, Desmon, and Vimentin have been shown to be cleaved directly by HIV protease, ${ }^{3-9}$ and when the eukaryotic initiation factor of translation (e1F4G-I) is directly cleaved by HIV protease, there is impaired translation of capped and uncapped mRNAs,, ,10,11 which may in turn lead to metabolic arrest, cellular respiration, and consequent death. Finally, studies derived from a cell-free system indicate that HIV protease may cleave procaspase 8 , resulting in a classical mitochondrial-dependent pathway of apoptosis involving Bid cleavage, mitochondrial depolarization, apoptosome formation, nuclear fragmentation, and PARP cleavage. $^{12}$

Importantly, the apoptotic pathology of HIV infection is complex, as cells directly infected with HIV, as well as cells that are not directly infected - succumb an apoptotic death. Virtually every HIV-encoded protein, in addition to HIV protease discussed above, has been suggested to modify host cell apoptosis, including gp120, gp160, Tat, Nef, Vpr, $\mathrm{Vpu}$, and Vif. Details of the effects of individual proteins on apoptosis during HIV is beyond the scope of this review, but in most cases, there impact is cell type specific, and in many cases these proteins can be proapoptotic or antiapoptotic depending on the context. ${ }^{13}$

\section{Role of HIV Protease Inhibitors in HIV Treatment}

The optimal current management paradigm for HIV disease is highly active antiretroviral therapy (HAART), which involves concurrent administration of multiple antiviral drugs that 
simultaneously target multiple aspects of the viral life cycle in order to both suppress viral replication and reduce the likelihood of generating viable escape mutants in treated individuals. HAART most often combines inhibitors of HIV reverse transcriptase with inhibitors of HIV protease. At present, there are eight currently approved protease inhibitors (PIs) (saquinivir, ritonavir, indinavir, nelfinavir, amprenavir, lopinavir, atazanivir, and fosamprenavir), and several others under development. The PI class of antiretroviral agents target the aspartyle protease of HIV, inhibiting the cleavage of viral polyproteins and the subsequent generation of individual viral proteins. This in turn prevents the formation of functional virions, although these drugs do not prevent upstream infection events, including entry reverse transcription and integration.

The introduction of HAART has been associated with dramatically reduced morbidity and mortality in patients infected with HIV, and has led to both quantitative and qualititative improvements in host immune function. The advent of Pl-based antiretroviral agents stimulated considerable interest in evaluating changes in the CD4 T-cell kinetics, in particular, whether the principal cause of CD4 depletion is impaired production or enhanced destruction. Recent data from two independent groups using independent approaches each conclude that the principal cause of CD4 T-cell decline is enhanced T-cell death, rather than impaired production. Since both studies ${ }^{14,15}$ showed that T-cell production rates in HIV infection actually increases, the degree of T-cell destruction is likely greater than that previously believed, as CD4 T-cell numbers decline even in the face of increased CD4 T-cell production rates. Moreover, T-cell production rates are highest in patients with the highest viral burdens, and initiation of antiretroviral therapy causes marked reductions in T-cell proliferative responses seen within 1-12 weeks. The changes that occur in naïve CD4 T-cell proliferation rates correlate with increases in the levels of $\mathrm{T}$-cell receptor excision circles. Finally, the reduced proliferation which is seen in response to HAART is lost following HAART cessation. ${ }^{16}$

In HIV-infected patients who are not receiving antiretroviral therapy, CD4 T-cell numbers continue to decline gradually over the course of the disease. Numerous studies, both in vitro and in vivo, indicate that enhanced apoptosis is a dominant mechanism for this CD4 T-cell loss. Such apoptosis occurs both within cells directly infected with HIV, as well as in those cells that are uninfected with HIV through bystander mechanisms (reviewed in Phenix et al. ${ }^{17}$ ). The magnitude of the CD4 T-cell apoptosis occurring in untreated patients appears to correlate directly with levels of immune activation, degree of viral replication, and stage of HIV disease (reviewed in Gougeon ${ }^{18}$ ). This enhanced lymphocyte apoptosis can be directly demonstrated in peripheral blood lymphocytes from HIV-infected patients when examined ex vivo, as either fresh samples, samples that have been cultured overnight, or samples that have been stimulated with either apoptosis inducing ligands, mitogen, or T-cell receptor ligation. In all situations, cells from HIV-infected patients have exaggerated apoptotic responses compared to cells from HIV uninfected patients. Not only is enhanced CD4 and CD8 T-cell apoptosis present in the peripheral blood compartment but also within lymphoid tissues (reviewed in Badley et al. ${ }^{19}$ ).
Since PI-based HAART dramatically increases CD4 T-cell numbers, it became of interest to determine what, if any, effect such therapies have on CD4 T-cell apoptosis. With remarkable consistency, numerous studies have now demonstrated that CD4 T-cell apoptosis is dramatically reduced in response to $\mathrm{PI}$-based therapy. Such reductions are observed in the peripheral blood, in cells cultured in vitro either in the presence or absence of activating and/or apoptotic stimuli, as well as in the lymphatic compartments. Such reductions in apoptosis are accompanied with reductions in HIV viral burden, decreases in HIV-associated immune activation, and improvements in patient's health (reviewed in Phenix et al. ${ }^{17}$ ). Moreover, where there is poor CD4 T-cell recovery persistently high levels of apoptosis are maintained. ${ }^{20}$

The mechanisms underlying the reduction in exaggerated apoptosis that follow HAART are almost certainly due in part to reductions in viral burden that result from treatment. Indeed, reductions in apoptosis are seen in patients who received non-PI-based therapies, indicating that reduced viral replication in and of itself is associated with reduced apoptosis. ${ }^{21}$ In addition, however, it is possible that PIs may possess intrinsic antiapoptotic properties, and a variety of indirect evidences suggest that this is the case:

1. CD4 T-cell numbers increase more with PI-based HAART compared to treatment that does not use $\mathrm{PI}$, even when with equivalent viral suppression. ${ }^{22,23}$

2. PI-based therapy may cause CD4 T-cell increase despite failure to suppress HIV viremia significantly. ${ }^{24,25}$

3. Regimens containing PI produce a greater CD8 T-cell increase than therapies using reverse transcriptase inhibitors alone. ${ }^{26}$

4. CD4 T-cell number continues to increase in patients with suppressed viral replication who continue PIs compared to those who switch off $\mathrm{PI}$ to start nonnucleoside-based reverse transcriptase inhibitors. ${ }^{27}$

5. Apoptosis is reduced following PI monotherapy, even in the absence of significant antiviral effect. ${ }^{28}$

6. Significant CD4 T-cell increases following PI-containing regimen compared to non- $\mathrm{Pl}$-containing regimens, even in the face of inferior antiviral outcomes ${ }^{29-31}$ (see Figure 1).

This abundant indirect data suggest that Pl-based therapy may result in improved CD4 T-cell reconstitution compared to non-PI-containing antiretroviral therapy. In a study designed to assess this question directly, $55 \mathrm{HIV}$-infected patients who were naïve to prior antiretroviral therapy were randomized to receive lopinavir/ritonavir, and nevaripine ( $\mathrm{PI}$ containing) or lamivudine, stavudine, abacavir, plus nevaripine (PI sparing). Viral load reductions were comparable between groups at weeks 8 and 48. An 8-week follow-up demonstrated similar CD4 T-cell change, CD8 T-cell change, as well as no differences in lymphocyte subset analyses comparing naïve and memory T-cell subsets. On the basis of these findings, the authors conclude that CD4 T-cell restoration is comparable between $\mathrm{Pl}$-containing versus $\mathrm{PI}$-sparing antiretroviral regimens. However, also included in that manuscript is a comparison of spontaneous apoptosis between groups. In the PI-sparing treatment arm, spontaneous apoptosis reduces gradually during the course of therapy. In contrast, 
in the PI-containing treatment arm, apoptosis is dramatically reduced after 1 week of treatment, a change which is not observed in the NRTI arm. ${ }^{32}$ Of note, this dramatic reduction in apoptosis that is seen prior to significant reduction in viral burden is entirely consistent with previous publications in a cohort of nonrandomized patients ${ }^{28}$ where dramatic reductions in apoptosis are seen within the first 4 days of treatment.
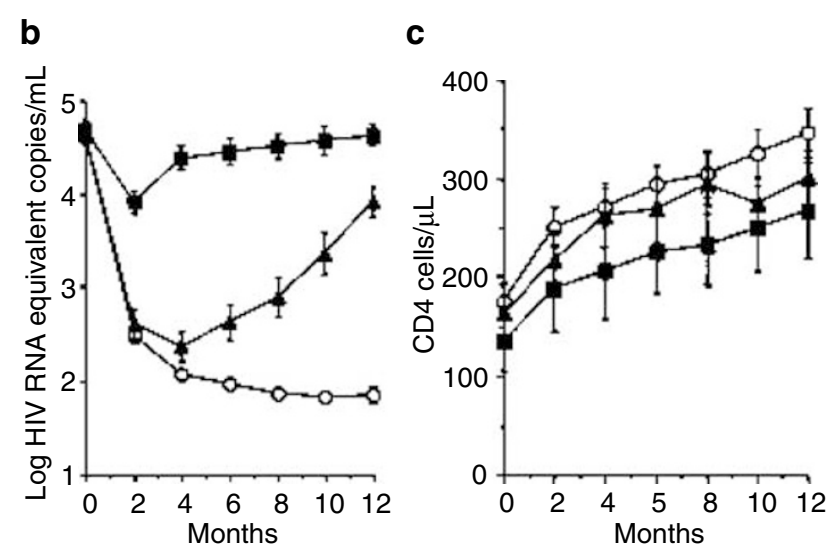

Figure 1 A total of 99 HIV seropositive, PI naïve, adults were treated with one or two PI and two nucleoside reverse transcriptase inhibitors and followed for 12 months. Every 2 months, patients blood was analyzed for viral replication and for CD4 T-cell count. Patients were classified as virologic responders (white circles), virologic nonresponders (black squares), or virologic transient responders (black triangles). CD4 T-cell recovery was comparable between groups, indicating a lack of dependence on viral suppression. Reprinted with permission. This research was originally published in Lu et al. HIV protease inhibitors restore impaired T-cell proliferative response in vivo and in vitro: a viral-suppressionindependent mechanism. Blood 2000; 96(1):250-258. ${ }^{(C)}$ The American Society of Hematology

\section{Mechanisms of PI-Mediated Apoptosis Inhibition}

At least five distinct mechanisms have been proposed to account for the antiapoptotic effects of PI (Figure 2).

\section{Decreased expression of apoptosis regulatory molecules}

One of the first papers to demonstrate reduced apoptosis following PI treatment in vitro, ${ }^{33}$ demonstrated convincingly that addition of ritonavir to hematopoietic colony-forming units augmented colony-forming unit replication; in cultures from HIV-infected patients, the addition of ritonavir resulted in a four-fold increase of both myeloid and erythroid colonies. To assess the putative mechanisms behind such reduced apoptosis and improved survival, the authors measured the expression of caspase 1 to determine whether the caspase transduction cascade was altered by ritonavir. In CD34positive hematopoietic progenerative cells, caspase 1 appears to be important and necessary for various forms of apoptosis. ${ }^{34}$ In that study, ritonavir was found to reduce the expression of caspase 1 as measured by intracellular flow cytometry, prompting the suggestion that Pls may modify apoptosis by virtue of altered expression, at either the transcriptional or translational level of caspase 1 or potentially other caspases. In a conceptually similar study, it has also been reported that membrane expression of Fas ligand in PHA-stimulated peripheral blood lymphocytes is decreased by ritonavir. ${ }^{35}$ In another study, the effect of saquinivir on anti-Fas- or anti-CD3-induced death of PBMCs from healthy donors was investigated. Pretreatment with drug for 4 days reduces susceptibility to both Fas-induced and

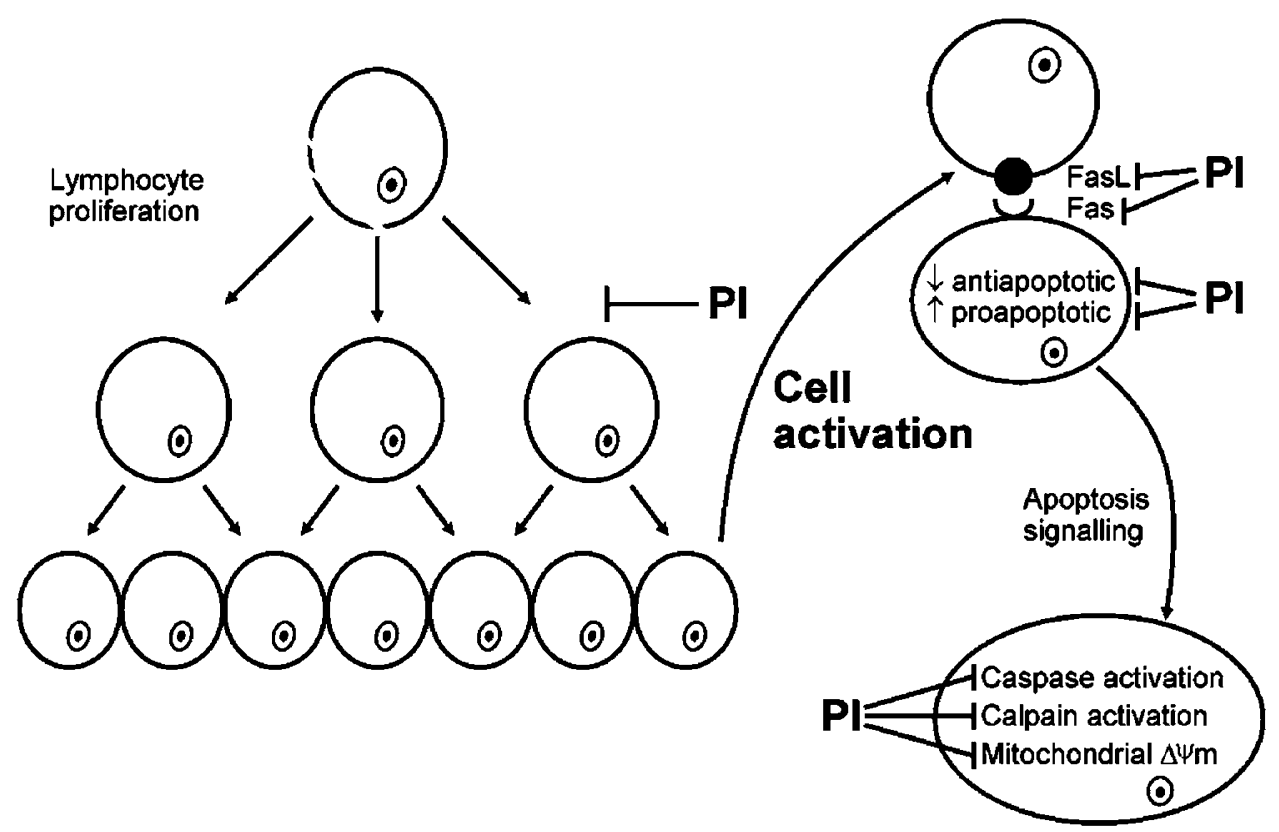

Figure 2 Conceptual model of proposed mechanisms by which PI inhibit apoptosis 
anti-CD3-induced apoptosis. This effect occurred in addition to the antiapoptotic effects of interleukin-2 (IL-2) treatment, and was associated with a decreased expression of Fas-receptor. The authors conclude that a potential mechanism of apoptosis inhibition is the reduced CD95 expression. ${ }^{36}$

Subsequent investigation does not support the model that $\mathrm{PI}$ are antiapoptotic by virtue of reduced expression of apoptosis regulatory molecules. Inhibition of protein synthesis by cyclohexamide did not reduce the ability of nelfinavir to inhibit camphothecin or Fas-induced apoptosis of Jurkat $T$ cells. ${ }^{37}$ Moreover, the effect of nelfinavir on mRNA or protein level for a variety of apoptosis regulatory proteins was not altered in these experiments. Similarly, subsequent work using saquinavir, indinavir, or lopinavir treatment of activated $T$ cells reduced apoptosis but did not alter intracellular levels a $\mathrm{BCL}-2, \mathrm{Bax}, \mathrm{BCL} \mathrm{xL}_{\mathrm{L}}$, or HSP-70 as assessed by intracellular flow cytometry. ${ }^{38}$

\section{Caspase inhibition}

An alternative model to account for the observed inhibition of apoptosis by HIV PIs suggests that caspase family members may be directly or indirectly inhibited by such drugs. ${ }^{35,39}$ Indeed, this proposal is intuitively appealing since HIV PIs themselves are likely to inhibit not only the aspartyle protease of HIV but also other cellular proteases. This model has been widely adopted by the HIV research community. Of note, however, is the observation that HIV protease itself is an aspartyle protease whereas caspases are cystine proteases, raising concerns as to the accuracy of this model from a stoichiometric perspective. At least three publications have directly assessed the impact of HIV PIs on caspase activity. Recombinant active caspases $1,3,6,7$, and 8 were incubated in the presence or absence of nelfinavir, vehicle control, or z-VAD-Fmk, in the presence of a fluorogenic caspase-specific substrate. In those experiments, addition of nelfinavir did not alter the ability of recombinant active caspases $1,3,6,7$, or 8 to cleave their respective fluorogenic substrates while nelfinavir maintained the ability to block HIV proteasemediated cleavage of a fluorogenic gag/pol consensus site. ${ }^{37}$ Such results have been reproduced using recombinant caspases 3, 6, and 8, incubated in the presence of indinavir or ritonavir where the activity of these caspases is not altered by either drug. ${ }^{40}$ Finally, the ability of indinavir to inhibit active caspases generated in vivo has also been assessed. Jurkat T-cells were stimulated to undergo apoptosis by the Fas agonists $\mathrm{CH}-11$ and cell lysates prepared. Caspase-specific fluorogenic substrate for caspases $1,3,4,5,8$, and 9 were added to cell lysates in the presence or absence of indinavir and, consistent with previous observations, the activity of these caspases was unaltered by indinavir but potently inhibited by peptidomimetic caspase inhibitors. ${ }^{41}$

\section{Altered proliferation}

A third model of how Pls may alter cell survival suggests that PIs themselves may alter proliferative responses of lymphocytes in vitro. Since the principal observation which spurred the suggestion that PIs might be antiapoptotic is the improved CD4 T-cell number seen in patients on PI-based therapies, this model would argue that T-cell increases are a result of enhanced proliferation as opposed to impaired death. Data that indirectly supports this model were presented in one of the first publications to describe the immunomodulatory effects of HIV PIs. Normal CD4 T cells were activated with IL-2 and PHA and then exposed to a Fas agonist. These cells showed increased viability and decreased apoptosis in the presence of ritonavir. $^{35}$ Other investigators subsequently directly assessed such a possibility. Peripheral blood mononuclear cells (PBMCs) from HIV-infected but untreated patients were harvested and incubated with indinavir or saquinivir and subsequently treated with PMA and anti-CD3 antibodies. Cells were then cultured in vitro with new PI added at each passage, and cumulative cell number monitored until day 16. The addition of PI dramatically enhanced cumulative cell number in this patient population, as well as in cells from HAART-treated patients. Importantly, such improved cell proliferation occurred even in those patients who did not manifest a virologic response to antiretroviral therapy. ${ }^{31}$ Such observations prompted the speculation that PIs enhanced CD4 T-cell number by virtue of improving proliferative responses, and since these authors did not observe modified apoptotic responses to TCR or Fas ligation-induced apoptosis by HIV PIs that the effect on CD4 T-cell number is solely an effect on proliferation as opposed to apoptosis inhibition. However, Chavan and colleagues assessed lymphoproliferation in PBMCs from healthy human volunteers precultured in media alone or media supplemented with different concentrations of indinavir followed by anti-CD3, PHA, Con-A, or PMA plus ionomycin. In these studies, indinavir resulted in a dosedependent reduction of proliferative responses to all stimuli tested. Moreover, such treatments led to cell cycle arrest in the G-0 or G-1 phase. ${ }^{41}$ Consistent with the suggestion that PIs may inhibit lymphoproliferative responses, another study examined foot pad swelling of C57BL6 mice infected with lymphocytic choriomeningitis virus (LCMV). Foot pad swelling reactions were markedly inhibited by ritonavir treatment in a dose-dependent manner, and this effect was conserved among a variety of different virus strains, different mouse strains, and both by parenteral and oral administration of the drug. ${ }^{42}$ These authors conclude that ritonavir (and potentially other PIs) may prolong cell survival indirectly by inhibiting their entry into cell cycle and thereby modifying the baseline susceptibility towards apoptotic signaling.

\section{Calpain inhibition}

Since apoptosis is commonly associated with abnormal calpain activation, it has also been suggested that HIV PIs may modify apoptotic signaling by virtue of direct inhibition of calpain. ${ }^{43,44}$ Using indinavir and ritonavir, apoptosis induced by purimyocin or etoposide in U937 cells was investigated. These authors found no impact of these drugs on caspase activity, and therefore analyzed calpain activity in lysates from U937 cells treated with these agents. Using a fluorogenic substrate assay, calpain-like activity was reduced by incubation of U937 cells with indinavir and ritonavir. Moreover, it has been described that indinavir and ritonavir directly inhibited recombinant $\mu$ calpane and $\mathrm{M}$ calpane. ${ }^{40}$ However, results from a different lab failed to demonstrate an effect of ritonavir 
on either autolytic or catalytic activity of $\mu$ calpain or $\mathrm{M}$ calpain. ${ }^{45}$ Other investigators have also reported in vitro results that ritonavir can inhibit calpains. ${ }^{46}$

\section{Inhibited loss of mitochondrial transmembrane potential $\left(\Delta \Psi_{m}\right)$}

A final proposed mechanism by which HIV PIs might inhibit apoptotic signaling involves prevention of mitochondrial transmembrane potential loss. Over the past decade, the role of mitochondria in apoptosis has become better defined, and it is now generally agreed that mitochondria serve as central regulators that coordinate the initiation phase with the executionary phases of apoptosis. Mitochondria serve as regulatory checkpoints for apoptotic signaling and, in fact, numerous apoptotic regulatory proteins including BCL-2 family members, IAPs, and SMAC/DIABLO function at the level of mitochondria to modify apoptotic responses. The first paper to evaluate the impact of PIs on mitochondrial apoptotic signaling performed multiparameter flow cytometry of Jurkat T cells stimulated with actinomycin $\mathrm{D}$, campothecin, or $\mathrm{CH}-11$ in the presence or absence of nelfinavir. These studies demonstrated that nelfinavir-treated cells stimulated to undergo apoptosis have less loss of mitochondrial transmembrane potential than cells treated with control agents. Moreover, mitochondrial release of cytochrome $c$ was inhibited in nelfinavir pretreated cells compared to control. Such observations that prompted the speculation that PIs might act at the level of mitochondria were then directly tested in two independent models. First isolated mitochondria were treated with nelfinavir and stimulated with the direct mitochondrial apoptotic agents atractyloside or VPR, demonstrating that nelfinavir but not vehicle control reduced mitochondrial transmembrane potential in these mitochondria. Further, nelfinavir was shown to inhibit mitochondrial-dependent apoptotic signaling but not mitochondrial-independent signaling. ${ }^{37}$

A second group evaluated the impact of PI on mitochondrial function by treating resting lymphocytes with lopinavir, indinavir and saquinivir, and stimulating the cells with antiFas, TNF- $\alpha$, TRAIL or UV irradiation, and measuring mitochondrial transmembrane potential. PI pre-exposure exerted a significant protection from $\Delta \Psi_{\mathrm{m}}$. To confirm this observation, since mitochondrial hyperpolarization has been related to reactive oxygen intermediate (ROI) hyperproduction, these investigators measured ROI production from cells stimulated in such a manner. All drugs tested significantly reduced the $\mathrm{ROI}$ production. These observations were supported by observations that PI prevent AZT (a mitochondrio-toxic HIV drug)-induced apoptosis of lymphocytes, and that PIs reduce caspase 9 and 2 activity, but not caspase 8 activity. ${ }^{38}$ Altogether, these studies suggest that PIs exert their antiapoptotic effect at a mitochondrial location, but the specific mitochondrial target is unknown.

\section{Effects of HIV PIs on Apoptosis In Vivo in Non-HIV Models}

Most studies that evaluate changes in apoptosis in response to antiretroviral therapies are confounded by at least two factors. First, the majority of these studies evaluate apoptosis changes that occur in patients receiving multiple anti-HIV drugs, commonly one or two Pls in addition to one or two reverse transcriptase inhibitors. Secondly, most of these studies evaluate changes in apoptosis that occur in HIV-infected patients, in whom a decrease in viral replication occurs almost universally, although the magnitude of these viral reductions is highly variable. Since reduced viral burden is likely to impact apoptosis, one cannot confidently ascribe reduced apoptosis solely either to direct drug effect or to reduced virus burden.

In light of these confounders, interest has developed in evaluating changes in apoptosis that occur in systems where HIV is not involved. One such study evaluated two HIV uninfected volunteers who received indinavir daily for 30 days. In both patients, shortly following the initiation of indinavir treatment, CD4 T-cell numbers dropped from baseline levels of approximately $1100 \mathrm{cells} / \mu \mathrm{l}$ to untreatment levels of approximately 900 cells/ $\mu$ l. Apoptosis itself was not measured in that study. ${ }^{47}$ More recently, this question has been readdressed. Eight healthy health-care workers with potential exposure to HIV were initiated on antiretroviral postexposure prophylaxis consisting of AZT, 3TC, and nelfinavir. Cells were harvested before therapy, during therapy, and at least 1 month after postexposure prophylaxis ended. Peripheral blood T-cell number and percentage did not change, nor did proliferative responses to mitogen or recall antigen. However, apoptosis susceptibility in response to campothecin was significantly reduced during antiretroviral therapy. Changes in Fas-induced apoptosis were observed, yet, due to the low level of Fasinduced killing these changes did not reach significance. ${ }^{48}$

Since disordered apoptosis is involved in the pathogenesis of numerous disease states in addition to HIV, we have recently evaluated the effects of $\mathrm{PI}$ therapy in a murine model of sepsis. Sepsis is associated with extensive lymphocyte and intestinal epithelial cell apoptosis, both in animal models, as well as in patients with sepsis (reviewed in Hotchkiss et al. ${ }^{49}$ ), and inhibition of apoptosis with pan caspase inhibitors significantly improves survival from sepsis in murine models. ${ }^{50}$ Given differences in drug metabolism between mouse and human, in order to achieve pharmacologically relevant serum levels of nelfinavir in mice, it was necessary to co-dose nelfinavir with ritonavir. Crude 48-h survival rates in mice treated with vehicle control was $17 \%$ compared to $67 \%$ in mice that received nelfinavir/ritonavir prior to cecal ligation and perforation, and $50 \%$ when nelfinavir/ritonavir treatment was begun $4 \mathrm{~h}$ following sepsis. Improved survival was correlated with a reduction in lymphocyte apoptosis, and an improvement in proinflammatory cytokine production (see Figure 3). ${ }^{51}$

\section{Paradoxical Pro-Apoptotic Effects of HIV PIs}

In addition to the well-described antiapoptotic effects of HIV PIs, an emerging body of literature suggests that these drugs may also be proapoptotic. Indeed, while HIV PIs appear to be antiapoptotic at concentrations of drug as low as $10 \mathrm{nM}$ (AD Badley, unpublished observations), whereas at significantly higher doses they may paradoxically induce apoptosis; treatment of monocytes and CD4 T cells with $10 \mu \mathrm{M}$ indinavir 


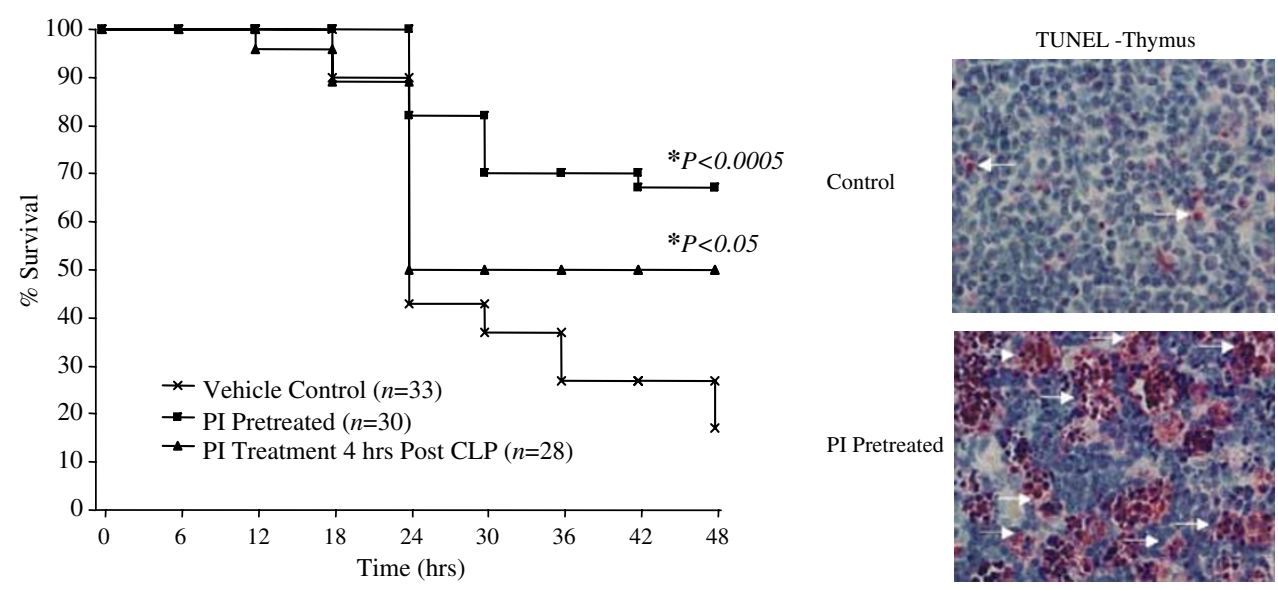

Figure 3 Female ND4 mice were anesthetized, the cecum externalized, punctured (cecal ligation and perforation, CLP), and replaced into the peritoneum. Mice received control vehicle gavage, or PI (nelfinavir/ritonavir) either before or after the CLP as indicated. Mice were followed for $48 \mathrm{~h}$, and survival monitored. In separate mice, $12 \mathrm{~h}$ following CLP, thymus was harvested and analyzed by TUNEL for apoptosis. Reprinted with permission. This research was originally published in Weaver et al. Improved survival in experimental sepsis with an orally administered inhibitor of apoptosis. The FASEB Journal 2004; 18:1185-1191
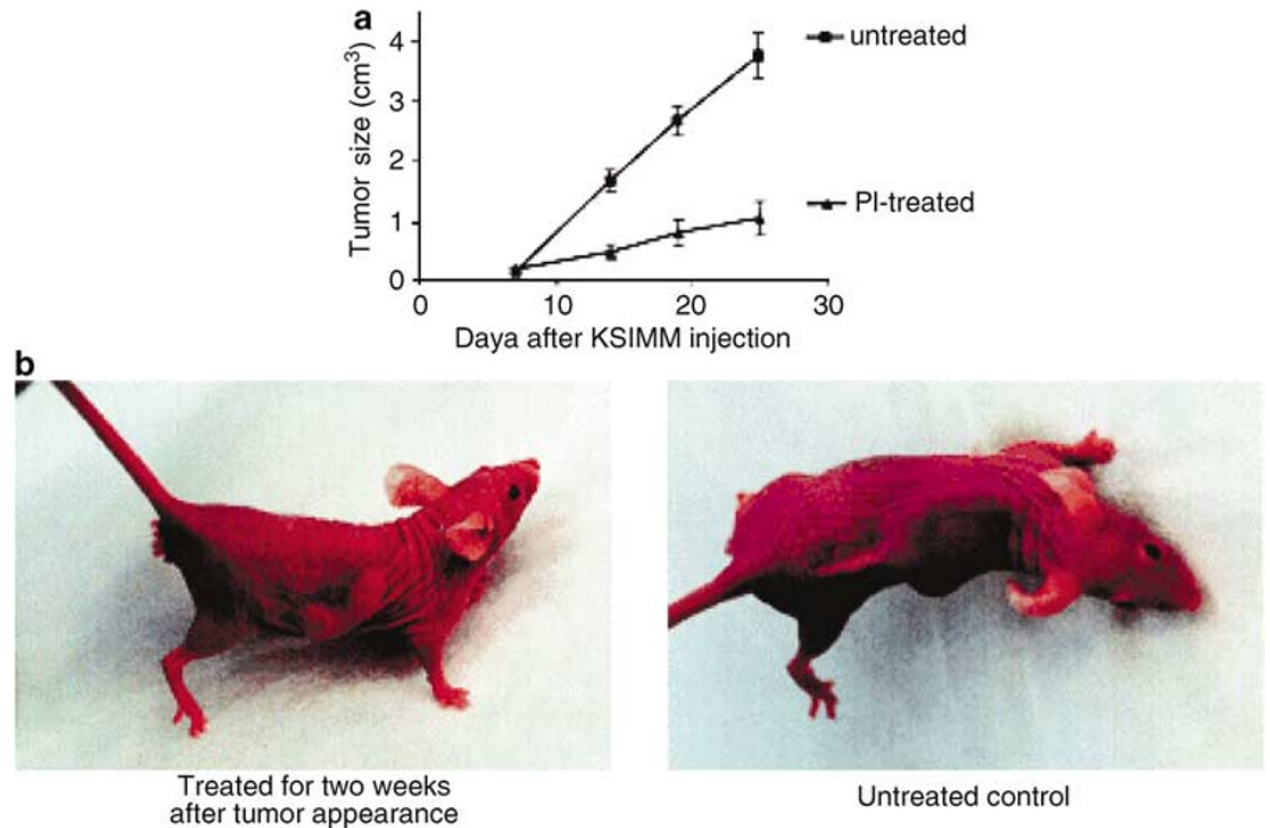

Figure 4 Male BNX mice were implanted with the KSIMM Kaposi's sarcoma cell line, and treated with ritonavir as indicated. Tumor size was monitored biweekly. Reprinted with permission. This research was originally published in Pati et al. Antitumorigenic effects of HIV protease inhibitor ritonavir: inhibition of Kaposi's sarcoma. Blood. 2002; 99(10): 3771-3779. ${ }^{\text {C }}$ The American Society of Hematology

or saquinivir significantly enhances the proportion of cells that bind annexin V-FITC. Moreover, we have observed enhanced apoptosis in Jurkat T-cell lines incubated with doses of lopinavir, ritonavir, and nelfinavir that exceed $10 \mu \mathrm{M}$ (AD Badley, unpublished data).

Consistent with the proposed proapoptotic effects of HIV PIs, several groups have evaluated the effect of ritonavir on tumor cell viability. Since prior publications have indicated that the HIV PI ritonavir can inhibit the peptidase activities of the $20 \mathrm{~S}$ proteosome, ${ }^{42}$ and since other proteosome inhibitors are both antiproliferative and induce apoptosis of tumor cells, ${ }^{52}$ the ability of ritonavir to induce tumor cell apoptosis was investigated. Using doses of ritonavir between 15 and $50 \mu \mathrm{M}$, a dose-dependent death of transformed cell lines characterized by intranucleosomal DNA laddering was observed. In contrast, nontransformed murine cells did not demonstrate DNA laddering in response to these doses of ritonavir. Furthermore, administration of $6 \mathrm{mg}$ of ritonavir per day to C57BL6 mice implanted with EL4 thymomas resulted in 76$79 \%$ growth inhibition of the thymoma compared to control animals. ${ }^{53}$ Of note, this dose of ritonavir is roughly equivalent to $300 \mathrm{mg} / \mathrm{kg} /$ day, whereas human dosing of ritonavir is approximately $17 \mathrm{mg} / \mathrm{kg} /$ day for a $70 \mathrm{~kg}$ patient. In the same publication, cells from treated animals had an accumulation of 
cells arrested in G1 phase that expressed high levels of P21 WAF1. Since P21 may be proapoptotic in different contexts, ${ }^{54}$ the authors speculate that the accumulation of P21 in the presence of ritonavir is likely due to inhibited proteosome activity, and that P21 accumulation may contribute to proapoptotic effects of this treatment.

In a separate study, the antitumor effects of ritonavir on Kaposi's sarcoma (KS) cells were investigated. The theoretical basis for this study was not only the proteosome inhibitory effects of ritonavir, but also observations that KS lesions can improve significantly in patients treated with $\mathrm{PI}$-based regimens, even in the absence of a reduction in HIV viral loads. ${ }^{55}$ In vitro data demonstrated that ritonavir treatment of a KS cell line can induce caspase-3-independent apoptosis. However, similar treatment of primary human umbilical veins endothelial cells did not. Such treatments were also associated with reduced production of proinflammatory cytokines, including interferon- $\gamma$, TNF- $\alpha$, interleukin- $1 \beta$, and IL-6, as well as decreased expression of endothelial cell adhesion molecules ICAM-1, VCAM-1, and E-selectin. Finally, when the KS cell line is implanted into an immunodeficient BNX mouse, treatments with ritonavir at doses of $30 \mathrm{mg} / \mathrm{kg} /$ day for 15 days resulted in decreased tumor size in treated animals compared to controls (see Figure 4). ${ }^{56}$

It is apparent, therefore, that under certain circumstances, particularly with high-dose $\mathrm{PI}$, a paradoxical proapoptotic effect can be observed. While the mechanisms underlying this proapoptotic effect are undefined, it is theoretically possible that these drugs may possess both an agonistic activity at high dose, yet an antagonistic activity at low dose, potentially by interacting with the same apoptosis regulatory protein. Such a mixed agonist/antagonist effect has been well described in other circumstances. For example, raloxisene is a mixed estrogen agonist/antagonist, which coincidentally can induce TUNEL-positive apoptosis of selected transformed cell lines. ${ }^{57}$ Moreover, other estrogen agonists/antagonists may upregulate proteins involved in the control of cell proliferation, transcription, and survival in some contexts, but alternately upregulate proteins involved in apoptosis in other contexts; ${ }^{58}$ raising the possibility that $\mathrm{PI}$ may function in a similar manner.

\section{Acknowledgements}

ADB is supported by grants from the National Institutes of Health (R01 Al062261-01 and R01 Al40384).

\section{References}

1. Strack PR, Frey MW, Rizzo CJ, Cordova B, George HJ, Meade R, Ho SP, Corman J, Tritch R and Korant BD (1996) Apoptosis mediated by HIV protease is preceded by cleavage of Bcl-2. Proc. Natl. Acad. Sci. USA 93: 9571-9576

2. Korant BD, Strack PR, Frey MW and Rizzo CJ (1998) A cellular anti-apoptosis protein is cleaved by the HIV-1 protease. Adv. Exp. Med. Biol. 436: 27-29

3. Shoeman RL, Honer B, Stoller TJ, Kesselmeier C, Miedel MC, Traub P and Graves MC (1990) Human immunodeficiency virus type 1 protease cleaves the intermediate filament proteins vimentin, desmin, and glial fibrillary acidic protein. Proc. Natl. Acad. Sci. USA 87: 6336-6340

4. Tomasselli AG, Hui JO, Adams L, Chosay J, Lowery D, Greenberg B, Yem A, Deibel MR, Zurcher-Neely H and Heinrikson RL (1991) Actin, Troponin C, Alzheimer amyloid precursor protein and pro-interleukin 1-beta as substrates of the protease from human immunodeficiency virus. J. Biol. Chem. 266: 14548-14553

5. Adams LD, Tomasselli AG, Robbins P, Moss B and Heinrikson RL (1992) HIV1 protease cleaves actin during acute infection of human T-lymphocytes. AIDS Res. Hum. Retroviruses 8: 291-295

6. Shoeman RL, Sachse C, Honer B, Mothes E, Kaufmann M and Traub P (1993) Cleavage of human and mouse cytoskeletal and sarcomeric proteins by human immunodeficiency virus type 1 protease. Actin, desmin, myosin, and tropomyosin. Am. J. Pathol. 142: 221-230

7. Shoeman RL, Huttermann C, Hartig R and Traub P (2001) Amino-terminal polypeptides of vimentin are responsible for the changes in nuclear architecture associated with human immunodeficiency virus type 1 protease activity in tissue culture cells. Mol. Biol. Cell. 12: 143-154

8. Ohlmann T, Prevot D, Decimo D, Roux F, Garin J, Morley SJ and Darlix J-L (2002) In vitro cleavage of elF4GI but not elF4GIl by HIV-1 protease and its effects on translation in the rabbit reticulocyte lysage system. J. Mol. Biol. 318: 9-20

9. Shoeman RL, Hartig R, Hauses C and Traub P (2002) Organization of focal adhesion plaques is disrupted by action of the HIV-1 protease. Cell. Biol. Int. 26: 529-539

10. Ventoso I, Blanco R, Perales $C$ and Carrasco L (2001) HIV-1 protease cleaves eukaryotic initiation factor $4 G$ and inhibits cap-dependent translation. Proc. Natl. Acad. Sci. USA 98: 12966-12971

11. Perales C, Carrasco L and Ventoso I (2003) Cleavage of elF4G by HIV-1 protease: effects on translation. FEBS Lett. 533: 89-94

12. Nie Z, Phenix BN, Lum J, Alam A, Lynch DH, Beckett B, Krammer PH, Sekaly RF and Badley AD (2002) HIV-1 protease processes procaspase 8 to cause mitochondrial release of chtychrome $\mathrm{c}$, caspase cleavage and nuclear fragmentation. Cell Death Differ. 9: 1172-1184

13. Petit $F$, Arnoult $D$, Viollet $L$ and Estaquier $J$ (2003) Intrinsic and extrinsic pathways signaling during HIV-1 mediated cell death. Biochimie 85: 795-811

14. Mohri H, Perelson AS, Tung K, Ribeiro RM, Ramratnam B, Markowitz M, Kost R, Hurley A, Weinberger L, Cesar D, Hellerstein MK and Ho DD (2001) Increased turnover of T lymphocytes in HIV-1 infection and its reduction by antiretroviral therapy. J. Exp. Med. 194: 1277-1287

15. Kovacs JA, Lempicki RA, Sidorov IA, Adelsberger JW, Herpin B, Metcalf JA, Sereti I, Polis MA, Davey RT, Tavel J, Falloon J, Stevens R, Lambert L, Dewar $\mathrm{R}$, Schwartzentruber DJ, Anver MR, Baseler MW, Masur H, Dimitrov DS and Lane HC (2001) Identification of dynamically distinct subpopulations of T lymphocytes that are differentially affected by HIV. J. Exp. Med. 194: 1731-1741

16. Lempicki RA, Kovacs JA, Baseler MW, Adelsberger JW, Dewar R, Natarajan V, Bosche MC, Metcalf JA, Stevens R, Lambert LA, Alvord WG, Polis MA, Davey RT, Dimitrov DS and Lane HC (2000) Impact of HIV-1 infection and highly active antiretroviral therapy on the kinetics of CD4+ and CD8+ T cell turnover in HIV-infected patients. Proc. Natl. Acad. Sci. USA 97: 13778-13783

17. Phenix BN, Cooper $C$, Owen $C$ and Badley AD (2002) Modulation of apoptotis by HIV protease inhibitors. Apoptosis 7: 295-312

18. Gougeon M-L (2003) Apoptosis as an HIV stretegy to escape immune attack. Nat. Rev. Immunol. 3: 392-404

19. Badley AD, Pilon AA, Landay A and Lynch DH (2000) Mechanisms of HIV associated lymphocyte apoptosis. Blood 96: 2951-2964

20. Swiss HIV Cohort Study (2004) Persistent apoptosis in HIV-1-infected individuals receiving potent antiretroviral therapy is associated with poor recovery of CD4 T lymphocytes. J. Acq. Immu. Def. Synd. 36: 671-677

21. Wasmuth JC, Hackbarth F, Rockstroh JK, Sauerbruch T and Spengler U (2003) Changes of lymphocyte apoptosis associated with sequential introduction of highly active antiretroviral therapy. HIV Med. 4: 111-119

22. Kravcik S, Magill A, Sanghvi B, Ogden R, Cameron DW, Lewis R, Yu G and Badley AD (2001) Comparative CD4 T cell responses of protease inhibitor containing and sparing antiretroviral regimens. HIV Clin. Trials 2: 160-170

23. Staszewski S, Morales-Ramirez J, Tashima KT, Rachlis A, Skiest D, Stanford J, Stryker R, Johnson P, Labriola DF, Farina D, Manion DJ, Ruiz NM and Study 006 Team (1999) Efavirenz plus zidovudine and lamivudine, efavirenz plus indinavir, and indinavir plus zidovudine and lamivudine in the treatment of HIV-1 infection in adults. New Engl. J. Med. 341: 1865-1873

24. Meroni L, Varchetta S, Manganaro D, Gatti N, Riva A, Mondorte A and Galli M (2002) Reduced levels of CD4 cell spontaneous apoptosis in human immundeficiency virus-infected patients with discordant response to protease inhibitors. J. Infect. Dis. 186: 143-144 
25. Lecossier D, Bouchonnet F, Schneider P, Clavel F and Hance AJ Centre de Recherche Integre sur le VIH Bichat-Claude Bernard. (2001) Discordant increases in CD4+ T cells in human immunodeficiency virus-infected patients experiencing virologic treatment failure: role of changes in thymic output and T cell death. J. Infect. Dis. 183: 1009-1016

26. Carr A, Emery S, Kelleher A, Law M and Cooper DA. (1996) CD8+ lymphocyte responses to antiretroviral therapy of HIV infection. J Acq. Immun. Def. Synd. 13: $320-326$

27. Owen C, Kazim F and Badley AD (2004) Effect on CD4 T cell count of replacing protease inhibitors in patients with successful HIV suppression: a metaanalysis. AIDS 18: 693-695

28. Badley AD, Parato K, Cameron DW, Kravcik S, Phenix BN, Ashby D, Kumar A, Lynch DH, Tschopp J and Angel JB (1999) Dynamic correlation of apoptosis and immune activation during treatment of HIV infection. Cell Death Differ. 6: $420-432$

29. Collier AC, Coombs RW, Schoenfeld DA, Bassett RL, Timpone J, Barugh A, Jones M, Facey K, Whitacre C, McAuliffe VJ, Friedman HM, Merigan TC, Reichman RC, Hooper C, Corey L and AIDS Clinical Trials Group (1996) Treatment of human immunodeficiency virus infection with saquinavir, zidovudine, and zalcitabine. New Engl. J. Med. 334: 1011-1017

30. Hirsch M, Steigbigel R, Staszewski S, Mellors J, Scerpella E, Hirschel B, Lange J, Squires K, Rawlins S, Meibohm A, Leavitt R and Protocol 039 Study Group (1999) A randomized, controlled trial of indinavir, zidovudine, and lamivudine in adults with advanced human immunodeficiency virus type 1 infection and prior antiretroviral therapy. J. Infect. Dis. 180: 659-665

31. Lu W and Andrieu J-M (2000) HIV protease inhibitors restore impaired T-cell proliferative response in vivo and in vitro: a viral-suppression-independent mechanism. Blood 96: 250-258

32. Landay AL, Spritzler J, Kessler H, Mildvan D, Pu M, Fox L, O'Neil D, Schock B Kuritzkes D, Lederman MM and Group ACT (2003) Immune reconstitution is comparable in antiretroviral-naive subjects after 1 year of successful therapy with a nucleoside reverse-transcriptase inhibitor or protease inhibitorcontaining antiretroviral regimen. J. Infect. Dis. 188: 1444-1454

33. Sloand EM, Maciejewski J, Kumar P, Kim S, Chaudhuri A and Young N (2000) Protease inhibitors stimulate hematopoiesis and decrease apoptosis and ICE expression in CD34(+) cells. Blood 96: 2735-2739

34. Sloand EM, Young NS, Sato T, Kim S and Maciejewski JP (1998) Inhibition of interleukin-1 beta-converting enzyme in human hematopoietic progenitor cells results in blockade of cytokine-mediated apoptosis and expansion of their proliferative potential. Exp. Hematol. 26: 1093-1099

35. Sloand EM, Kumar PN, Kim S, Chaudhuri A, Weichold FF and Young NS (1999) Human immunodeficiency virus type 1 protease inhibitor modulates activation of peripheral blood CD4(+) T cells and decreases their susceptibility to apoptosis in vitro and in vivo. Blood 94: 1021-1027

36. Estaquier J, Lelievre J-D, Petit F, Brunner T, Moutouh-de Parseval L, Richman DD, Ameisen JC and Corbeil J (2002) Effects of antiretroviral drugs on human immunodeficiency virus type 1-induced CD4+ T-cell death. J. Virol. 76: 5966-5973

37. Phenix BN, Lum JJ, Nie Z, Sanchez-Dardon J and Badley AD. (2001) Antiapoptotic mechanism of HIV protease inhibitors: preventing mitochondrial transmembrane potential loss. Blood 98: 1078-1085

38. Matarrese P, Gambardella L, Cassone A, Vella S, Cauda R and Malorni W. (2003) Mitochondrial membrane hyperpolarization hijacks activated T lymphocytes toward the apoptotic-prone phenotype: homeostatic mechanisms of HIV protease inhibitors. J. Immunol. 170: 6006-6015

39. Weichold FF, Bryant JL, Pati S, Barabitskaya O, Gallo RC and Reitz MSJ. (1999) HIV-1 protease inhibitor ritonavir modulates susceptibility to apoptosis of uninfected T cells. J. Hum. Virol. 2: 261-269

40. Ghibelli L, Mengoni F, Lichtner M, Coppola S, De Nicola M, Bergamaschi A, Mastroianni C and Vullo V (2003) Anti-apoptotic effect of HIV protease inhibitors via direct inhibition of calpain. Biochem. Pharmacol. 66: 1505-1512
41. Chavan S, Kodoth S, Pahwa R and Pahwa S (2001) The HIV protease inhibitor indinavir inhibits cell-cycle progression in vitro in lymphocytes of HIV-infected and uninfected individuals. Blood 98: 383-389

42. Andre P, Groettrup M, Klenerman P, De Guili R, Booth BLJ, Cerundolo V, Bonneville M, Jotereau F, Zinkernagel RM and Lotteau V (1998) An inhibitor of HIV-1 protease modulates proteasome activity, antigen presentation, and T cell responses. Proc. Natl. Acad. Sci. USA 95: 13120-13124

43. Vanderklish PW and Bahr BA. (2000) The pathogenic activation of calpain: a marker and mediator of cellular toxicity and disease states. Int. J. Exp. Pathol. 81: $323-339$

44. Suzuki K, Hata S, Kawabata Y and Sorimachi H (2004) Structure, activation, and biology of calpain. Diabetes 53 (Suppl. 1): S12-S18

45. Cuerrier D, Nie Z, Badley AD and Davies PL (2005) Ritonavir does not inhibit calpain in vitro. Biochem. Biophys. Res. Commun. 327: 208-211

46. Wan W and DePetrillo PB (2002) Ritonavir inhibition of calcium-activated neutral proteases. Biochem. Pharmacol. 63: 1481-1484

47. Barker E, Kahn J, Fujimura S and Levy JA (1998) Protease inhibitors do not increase the CD4+ cell count in HIV-uninfected individuals. AIDS 12: $1117-1118$

48. Cooper CL, Phenix BN, Mbisa G, Lum JJ, Parato K, Nawley N and Angel JB andBadley AD. (2004) Antiretroviral therapy influences cellular susceptibility to apoptosis in vivo. Frontiers Biosci. 9: 338-341

49. Hotchkiss RS, Swanson PE, Freeman BD, Tinsley KW, Cobb JP, Matuschak GM, Buchman TG and Karl IE. (1999) Apoptotic cell death in patients with sepsis, shock, and multiple organ dysfunction. Crit. Care Med. 27: 1230-1251

50. Hotchkiss RS, Chang KC, Swanson PE, Tinsley KW, Hui JJ, Klender P, Xanthoudakis S, Roy S, Black C, Grimm E, Aspiotis R, Han Y, Nicholson DW and Karl IE. (2000) Caspase inhibitors improve survival in sepsis: a critical role of the lymphocyte. Nat. Immunol. 1: 496-501

51. Weaver JGR, Rouse MS and Steckelberg JM andBadley AD. (2004) Improved survival in experimental sepsis with an orally administered inhivitor of apoptosis. FASEB J. 18: 1185-1191

52. Orlowski RZ (1999) The role of the ubiquitin-proteasome pathway in apoptosis. Cell Death Differ. 6: 303-313

53. Gaedicke S, Firat-Geier E, Constantiniu O, Lucchiari-Hartz M, Freudenberg M, Galanos C and Niedermann G (2002) Antitumor effect of the human immunodeficiency virus protease inhibitor ritonavir: induction of tumor-cell apoptosis associated with perturbation of proteasomal proteolysis. Cancer Res. 62: 6901-6908

54. Sekiguchi T and Hunter T (1998) Induction of growth arrest and cell death by overexpression of the cyclin-Cdk inhibitor p21 in hamster BHK21 cells. Oncogene 16: 369-380

55. Lebbe C, Blum L, Pellet C, Blanchard G, Verola O, Morel P, Danne O and Calvo $F$ (1998) Clinical and biological impact of antiretroviral therapy with protease inhibitors on HIV-related Kaposi's sarcoma. AIDS 12: F45-F49

56. Pati S, Pelser CB, Dufraine J, Bryant JL, Reitz MSJ and Weichold FF (2002) Antitumorigenic effects of HIV protease inhibitor ritonavir: inhibition of Kaposi sarcoma. Blood 99: 3771-3779

57. Kim IY, Seong DH, Kim B-C, Lee DK, Remaley AT, Leach F, Morton RA and Kim S-J (2002) Raloxifene, a selective estrogen receptor modulator, induces apoptosis in androgen-responsive human prostate cancer cell line LNCaP through an androgen-independent pathway. Cancer Res. 62: 3649-3653

58. Levenson AS, Svoboda KM, Pease KM, Kaiser SA, Chen B, Simons LA, Jovanovic BD, Dyck PA and Jordan VC (2002) Gene expression profiles with activation of the estrogen receptor alpha-selective estrogen receptor modulator complex in breast cancer cells expressing wild-type estrogen receptor. Cancer Res. 62: 4419-4426 